\title{
Multiscale Modeling of Circular and Elliptical Particles in Laminar Shear Flow
}

\author{
Nenad Filipovic*, Velibor Isailović, Tijana Đukić, Mauro Ferrari, and Milos Kojic
}

\begin{abstract}
Drug delivery systems for cancer prevention and pain management have been improved related to classical cancer chemotherapy. Nanotechnology with nanoparticles offers new ways in transport of drug molecules and contrast agents by the blood flow through the circulatory system. In this study, we use multiscale mesoscopic bridging procedure of the finite elements (FE) coupled with dissipative particle dynamics (DPD) and lattice Boltzmann (LB) method to model the motion of circular and elliptical particles in a 2-D laminar flow. Four examples are considered: 1) one sedimenting cylinder in a channel, 2) two sedimenting cylinders in a channel, 3 ) motion of four elliptical particles in a linear shear flow, and 4) motion of circular and elliptical particle in the arterial bifurcation geometry. A good agreement with solution from the literature available was found. These results show that the multiscale approach with coupled FE and DPD/LB methods can effectively be applied to model motion of micro/nanoparticles for a drug delivery system.
\end{abstract}

Index Terms-Dissipative particle dynamics (DPD), finite element (FE), Lattice Boltzmann (LB) methods, margination, multiscale mesoscopic bridging, nanoparticles, 2-D laminar shear flow.

\section{INTRODUCTION}

I T IS WELL known that the efficacy of molecule-based therapies and imaging is mainly delivery limited. A multitude of barriers along the circulatory system prevent therapeutic molecules and tracers for imaging from reaching their biological target in the desired mass fractions [1]. The use of drug delivery systems has been improved in the areas of cancer prevention and pain management. Furthermore, the encapsulation of the drug in a microreservoir can improve drug solubility and stability, as well as reduce drug resistance, in some human carcinomas [2].

Nanotechnology offers opportunities to interface with biology in new ways and with unprecedented precision. The size of nanoparticles is becoming an important variable that affects the way they enter cells and how they influence the cell functions [3].

Manuscript received April 2, 2011; revised June 12, 2011; accepted August 13, 2011. Date of publication August 30, 2011; date of current version December 21, 2011. This work was supported by The Methodist Hospital Research Institute, Houston, TX. Asterisk indicates corresponding author.

*N. Filipovic and T. Đukić are with the University of Kragujevac, Kragujevac 34000, Serbia (e-mail: fica@kg.ac.rs; tijanakg@eunet.rs).

V. Isailović is with Bioengineering R\&D Center, Bioengineering Research and Development Center, Kragujevac 34000, Serbia (e-mail: velibor@kg.ac.rs).

M. Ferrari and M. Kojic are with The Methodist Hospital Research Institute, Houston, TX 77030 USA (e-mail: mferrari@tmhs.org; mkojic@hsph. harvard.edu).

Color versions of one or more of the figures in this paper are available online at http://ieeexplore.ieee.org.

Digital Object Identifier 10.1109/TBME.2011.2166264
There are a number of mathematical models regarding different aspects of the journey that nanoparticles have to take from the site of intravascular injection to the final biological target [4]-[6]. In this study, we proposed multiscale mesoscopic bridging method, where the whole fluid domain is divided into: 1) global domain, where only a continuum macroscopic model is used and 2) local domains, where both the macroscopic model and the mesoscopic discrete particle model are used, with the appropriate coupling between these models.

We examined particle motion in 2-D channel and motion of circular and elliptical particles in a linear shear flow. Two different methodologies to solve this problem were implemented. The finite-element (FE) and multiscale mesoscopic method for fluid-solid interaction was implemented in our in-house FE procedure [7]. dissipative particle dynamics (DPD) and lattice Boltzmann (LB) methods were applied for the mesoscopic domain and motion of the circular and elliptical particles in a linear shear flow. Finally, discussion and conclusions are described.

\section{MEthodS}

\section{A. Governing Equation for the Fluid Domain}

We here present the final form of equations for the fluid domain. The incremental-iterative balance equation of an FE in the standard Galerkin procedure for a time step " $n$ " and equilibrium iteration " $i$ " has a form [7]

$$
\begin{aligned}
& {\left[\begin{array}{cc}
\frac{1}{\Delta t} \mathbf{M}+{ }^{n+1} \tilde{\mathbf{K}}_{v v}^{(i-1)} & \mathbf{K}_{v p} \\
\mathbf{K}_{v p}^{T} & \mathbf{0}
\end{array}\right]\left\{\begin{array}{l}
\Delta \mathbf{V}^{(i)} \\
\Delta \mathbf{P}^{(i)}
\end{array}\right\}_{\text {blood }}=\left\{\begin{array}{c}
{ }^{n+1} \mathbf{F}_{\text {ext }}^{(i-1)} \\
0
\end{array}\right\}} \\
& -\left[\begin{array}{cc}
\frac{1}{\Delta t} \mathbf{M}+{ }^{n+1} \mathbf{K}^{(i-1)} & \mathbf{K}_{v p} \\
\mathbf{K}_{v p}^{T} & 0
\end{array}\right]\left\{\begin{array}{l}
{ }^{n+1} \mathbf{V}^{(i-1)} \\
{ }^{n+1} \mathbf{P}^{(i-1)}
\end{array}\right\} \\
& +\left\{\begin{array}{c}
\frac{1}{\Delta t} \mathbf{M}^{n} \mathbf{V} \\
0
\end{array}\right\}
\end{aligned}
$$

where ${ }^{n+1} \mathbf{V}^{(i-1)}$ and ${ }^{n+1} \mathbf{P}^{(i-1)}$ are the nodal vectors of fluid velocity and pressure, with the increments in the time steps $\Delta \mathbf{V}^{(i)}$ and $\Delta \mathbf{P}^{(i)}$ respectively; $\Delta t$ is the time step size and the left upper indices " $n$ " and " $n+1$ " denote the start and the end of time step; and the matrices and vectors are defined in [7]. The vector ${ }^{n+1} \mathbf{F}_{\text {ext }}^{(i-1)}$ of external forces includes the volumetric and surface forces. In assembling of these equations, the system of equations of the form (1) is obtained, with the volumetric external forces and the surface forces acting only on the fluiddomain boundary (the surface forces among the internal element boundaries cancel). 


\section{B. DPD Equations}

In the DPD model, a fluid is discretized into a set of interacting particles. Each DPD particle represents a cluster of molecular dynamics (MD) particles (atoms or molecules). There exists a mass fluctuation since MD particles can enter and go out from a Voronoi cell. Also, the shape of the cells changes as the cells move. However, this mass fluctuation and change of shape are neglected in the DPD formulation particles. Hence, the DPD particles representing the Voronoi cells have constant mass during motion.

The evolution of the particle position $\mathbf{r}_{i}$ can be obtained by the application of the Newton second law:

$$
\begin{aligned}
\dot{\mathbf{r}}_{i} & =\mathbf{v}_{i} \\
\dot{\mathbf{v}}_{i} & =\frac{1}{m_{i}} \sum_{j \neq i}^{N} \hat{\mathbf{F}}_{i j}=\sum_{j \neq i}^{N} \mathbf{F}_{i j}
\end{aligned}
$$

where $\mathbf{v}_{i}$ is the particle velocity; $m_{i}$ is the particle mass; $\hat{\mathbf{F}}_{i j}$ is the total force on particle $i$ due to particle $j$, while $\mathbf{F}_{i j}$ is the force per unit mass; and the dot indicates a time derivative. In our analysis, we assume that each particle has the same mass. Here, we have neglected the external forces for simplicity.

The interaction forces can be represented as the sum of three forces [7]: conservative (repulsion) $\mathbf{F}_{i j}^{C}$, dissipative $\mathbf{F}_{i j}^{D}$, and random force $\mathbf{F}_{i j}^{R}$ :

$$
\mathbf{F}_{i j}=\mathbf{F}_{i j}^{C}+\mathbf{F}_{i j}^{D}+\mathbf{F}_{i j}^{R} .
$$

\section{LB Method}

The equation that represents the LB numerical scheme and that is implemented in all the solvers based on the LB method is given by

$$
\begin{aligned}
f_{i}\left(\mathbf{x}+v_{i}, t+1\right)-f_{i}(\mathbf{x}, t)= & -\frac{1}{\tau}\left(f_{i}(\mathbf{x}, t)-f_{i}^{(0)}(\rho, \mathbf{u})\right) \\
& +\left(1-\frac{1}{2 \bar{\tau}}\right) F_{i} .
\end{aligned}
$$

In the implementation of this method, this equation is solved in two distinct steps: collision step and propagation step [8]. $f_{i}^{\text {in }}$ and $f_{i}^{\text {out }}$ are the values of the discretized distribution function $f_{i}$ before and after the collision and $f_{i}^{\text {out }}$ is, at the same time, the value of the distribution function that represents "input data" for the next time step.

The mentioned steps are now given as follows: the collision

$$
\begin{aligned}
f_{i}^{\text {out }}(\mathbf{x}, t)= & f_{i}^{\text {in }}(\mathbf{x}, t)-\frac{1}{\tau}\left(f_{i}^{\text {in }}(\mathbf{x}, t)-f_{i}^{(0)}(\rho, \mathbf{u})\right) \\
& +\left(1-\frac{1}{2 \bar{\tau}}\right) F_{i}
\end{aligned}
$$

and the propagation

$$
f_{i}^{\text {in }}\left(\mathbf{x}+v_{i}, t+1\right)=f_{i}^{\text {out }}(\mathbf{x}, t) .
$$

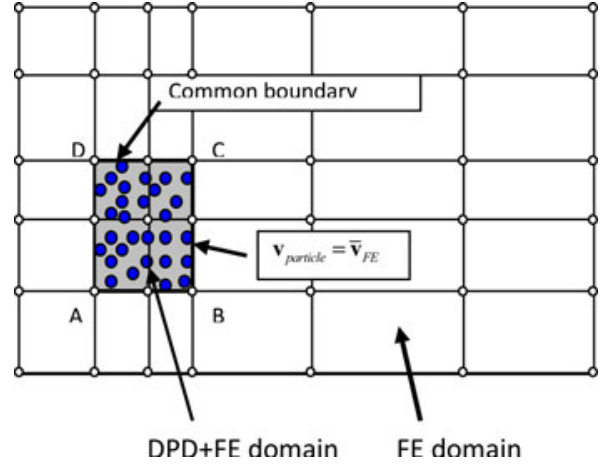

Fig. 1. Two domains within a flow field and boundary conditions at the common boundary between fine (meso) and coarse (FE) models [11].

\section{Multiscale Method}

For the multiscale method, we use similar methodology as the coupling between the MD and continuum models. It is according to the bridging scale (BS) method [9], [10]. The main coupling has been achieved through the interaction forces between the mesoscale particles.

We coupled the DPD/LB method with the corresponding nodal forces of an FE model. This methodology divides a fluid domain into the domains for a coarse scale modeling (only), and local domains modeled by both coarse scale (FE) and detailed mesoscale DPD/LB, with the coupling between the governing equations of the two (or multiple) different scales in a consistent manner. To enable full consistent coupling, the appropriate boundary conditions at the common boundary between the two domains have to be imposed. The fluid flow is modeled by FE in the whole domain and by the DPD discrete particles within the local domain as shown in Fig. 1. The flow region outside the local domain is called the global domain. The boundary between local and global domains is shown as the line ABCD. It is assumed that the boundary ABCD is fit along the lines of the FE mesh.

\section{RESULTS}

First, we performed multiscale simulation with FE-DPD and FE-LB of flow around a cylinder of the radius $R$, which is moving in a stagnant fluid with relative velocity $\mathbf{V}$. An analytical solution for the drag force $\mathbf{F}$ is equal to

$$
\mathbf{F}=-C_{D} \pi \rho R^{2}|\mathbf{V}| \mathbf{V}
$$

where $C_{D}$ is the drag coefficient. For Reynolds number Re, the drag coefficient becomes $C_{D}=\frac{24}{R_{e}}\left(1+(3 / 16) R_{e}\right)$ [12]. Equation (7) reduces to

$$
\mathbf{F}=6 \pi \mu R \mathbf{V}\left(1+\frac{3}{8} R_{e}\right) .
$$

The 2-D system of DPD and LB particles for simulation of the drag force, where the motion of a fluid past a stationary circular cylinder located at the center of the simulation box, was used. A constant force is applied to all fluid particles. The height of the simulation box is 40 times larger than the diameter of the disk. Periodic boundary conditions are used for input and 


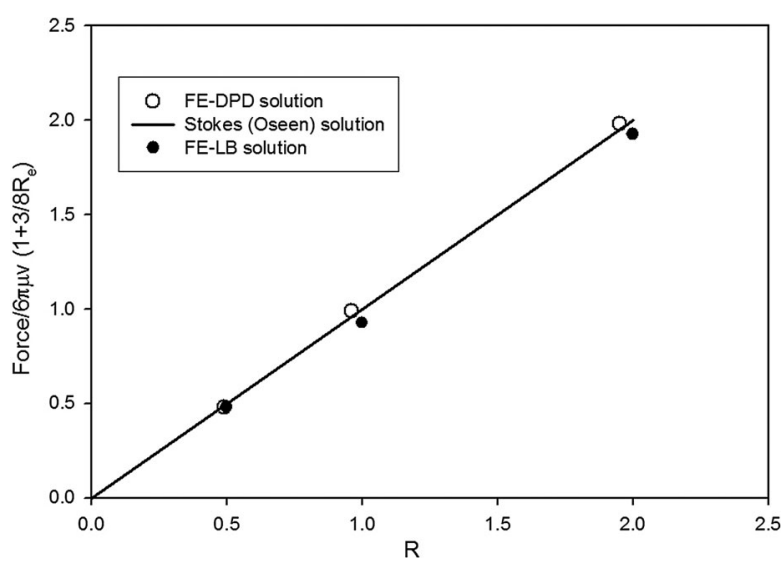

Fig. 2. Drag force as the function of circular cylinder radius $R$.

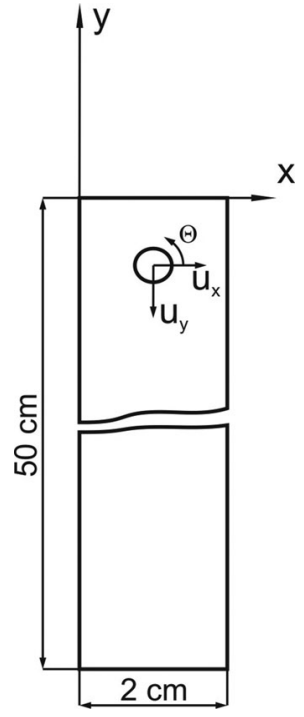

Fig. 3. Basic geometry data for the problem. One cylinder sedimenting in a channel.

output. There is no motion of the particles at the channel walls and those used to model the rigid cylinder.

The circular cylinder is modeled as the freezing group of the DPD and LB particles. The total force on the circular cylinder is calculated as sum of all DPD and LB forces on the freezing particles inside the cylinder. The linearity of the mean drag force and radius can be observed from Fig. 2, which corresponds to the Stokes (Oseen) regime and the analytical solution for the drag force [12].

The next example is related to the motion of one sedimenting cylinder in the channel, which is presented in Fig. 3 [13]. We used the multiscale FE-DPD procedure to solve this problem. The width of the channel is $4 d$, where $d=0.5 \mathrm{~cm}$ is the diameter of the cylinder. The cylinder starts at the center of the channel. The height of the channel is $50 \mathrm{~cm}$. The cylinder is falling with the gravity force. The density of the fluid is $\rho=1 \mathrm{~g} / \mathrm{cm}^{3}$ and dynamic viscosity is $1 \times 10^{-2} \mathrm{~g} / \mathrm{cm} \cdot \mathrm{s}$.

The diameter of cylinders is $d=0.5 \mathrm{~cm}$, diameters of the ellipsoids are $a=0.5 \mathrm{~cm}, b=0.25 \mathrm{~cm}$, and the width of the channel is $2 \mathrm{~cm}$.

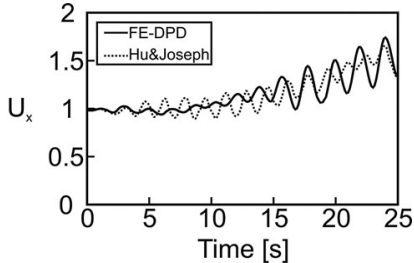

(a)

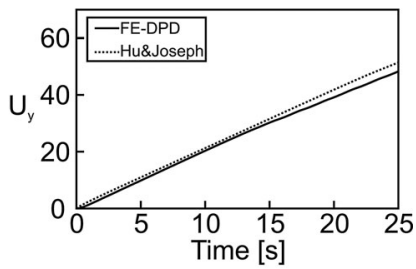

(b)

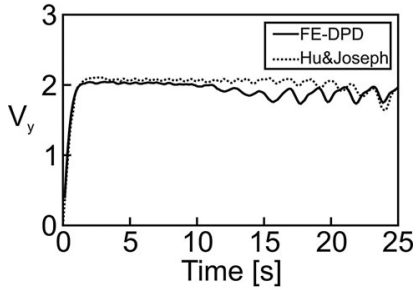

(c)

Fig. 4. One sedimenting cylinder in a channel. The diameter of the cylinder is $d=0.5 \mathrm{~cm}$ and the width of the channel is $2 \mathrm{~cm}$. The cylinder starts at the center of the channel. (a), (b), (c) Comparison of displacements and velocity of the cylinder in the $X$ and $Y$ directions with those in [13]. The length and velocity are nondimensionalized with $d$ being the diameter of cylinder and $V t$ the terminal velocity.

Displacements in time for the $X$ and $Y$ directions are presented in Fig. 4(a) and (b), respectively. Also, velocity distribution in the $Y$ direction is shown in Fig. 4(c). From these figures, it can be seen that dominant motion is the periodic oscillation, which is associated with vortex shedding behind the cylinder. Velocity distributions for one and two cylinders and four ellipsoids sedimenting in a channel are presented in Fig. 5. These results are in good agreement with [13]. Simulation time for these examples is around $200 \mathrm{~h}$ on ten Intel 2.4-GHz parallel processors.

The mesoscale domain of cylinder and ellipsoid was modeled with the DPD model, with the system containing $5000 \times 500$ DPD beads. The DPD particle distribution corresponded to the number density $n=4$ and the average distance between beads is $\lambda=1 / 3$. The DPD particles at the wall were assumed frozen, as well as particles inside the elliptic rigid particle. A linear shear flow was applied from the finite-element domain. The DPD parameters for fluid-fluid particles were $a_{i j}=25 k_{B} T$ and $\gamma=4.5 k_{B} T$, where $k_{B} T$ is assumed to be equal to unity. The fluid-particle interaction parameter was set to $a_{i j}=100 k_{B} T / \rho$. A constant time step $\Delta t=2 \times 10^{-4}$ (DPD time) was used for this simulation. The multiscale FE-DPD and FE solutions for the particle center trajectory are shown in Fig. 6 [14].

Trajectories of the circular and elliptical particles, which are moving in the simplified arterial bifurcation geometry, are shown in Fig. 7. At the inlet, a parabolic velocity profile is prescribed and is a free surface boundary condition at the 


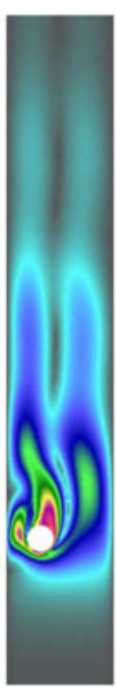

(a)

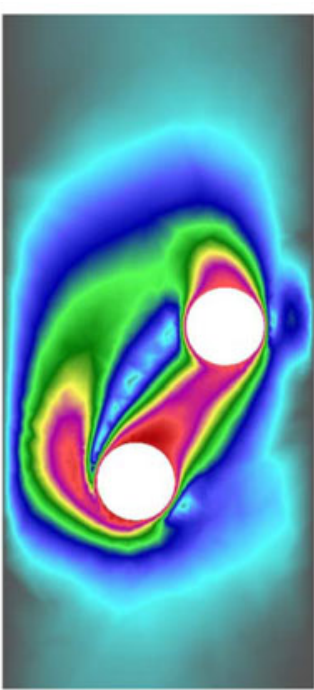

(b)

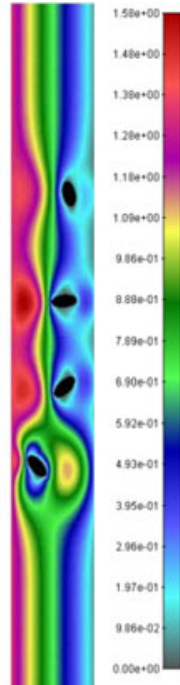

(c)
Fig. 5. Velocity distribution for (a) one cylinder, (b) two cylinders, and (c) four ellipsoids sedimenting in a channel.

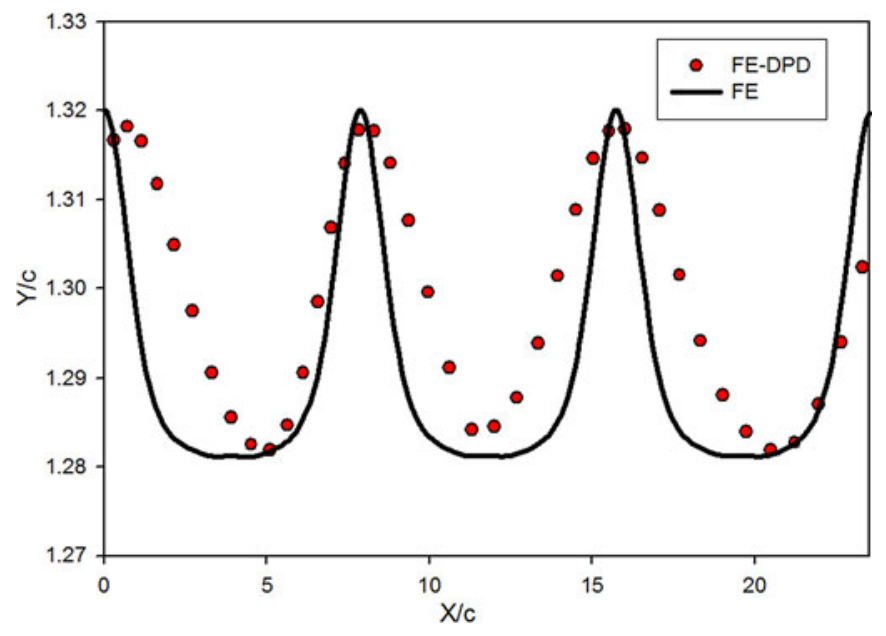

Fig. 6. Trajectory of an elliptical particle in a linear laminar flow with $\theta_{0}=$ $\pi / 2$ in the absence of gravitational force. The FE solution with $S t=0.125$ and multiscale FE-DPD solution with the shear external force $F_{x}=1$.

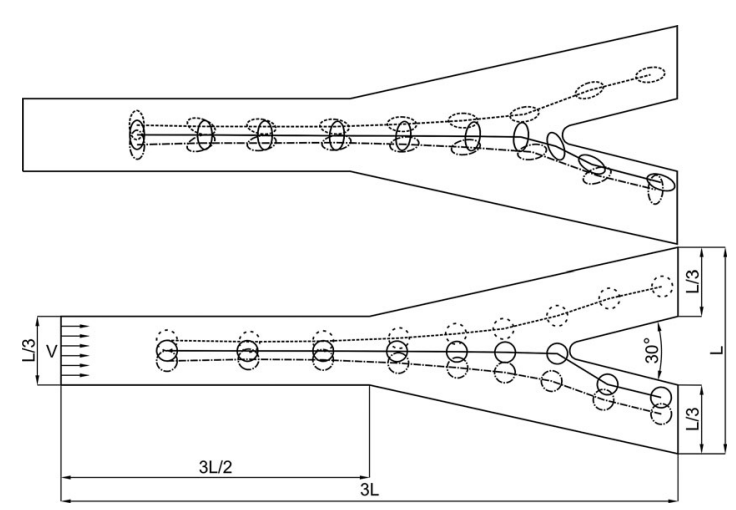

Fig. 7. Circular and elliptical particle motion in the simplified arterial bifurcation geometry. $L=6 \mathrm{~mm}, V=1 \mathrm{~mm} / \mathrm{s}$. Different trajectories are denoted with different line types: solid, short dash, and dash-dotted. outlet. The multiscale FE-DPD method was applied for the local circular- and elliptical-particle-moving domain.

\section{DISCUSSION}

DPD and LB methods were coupled with the continuum finite-element method as a multiscale mesoscopic bridging scale method. The fluid domain was divided into: 1) global domain, where only a continuum macroscopic model was used and 2) local domain, where both macroscopic model and mesoscopic discrete particle model were used.

We found good agreement between our results and the literature for one sedimenting cylinder motion in the channel. Also, the interaction between two cylinders and four ellipsoids shows drafting, kissing, and tumbling, which are a local rearrangement mechanism in a two-phase flow of solids and liquids in beds of particles confined to move in two dimensions. This multiscale approach is of particular interest in biomedical sciences, for investigations of blood flow within microvessels, cell or particle transport, and margination to the vessel walls and drug delivery.

\section{REFERENCES}

[1] P. Decuzzi, F. Causa, M. Ferrari, and P. A. Netti, "The effective dispersion of nanovectors within the microvasculature," Ann. Biomed. Eng., vol. 34, no. 4, pp. 633-641, 2006.

[2] Z. Reiner, R. Dominik, and F. Iduna F, "Reduction of tamoxifen resistance in human breast carcinomas by tamoxifen-containing liposomes in vivo," Anti-Cancer Drugs, vol. 15, pp. 707-714, 2004.

[3] G. Orive, R. M. Hernndez, A. R. Gascn, A. Dom£nguez-Gil, and J. L. Pedraz, "Drug delivery in biotechnology: Present and future," Current Opin. Biotechnol., vol. 14, pp. 659-664, 2003.

[4] P. Decuzzi, S. Lee, B. Bhushan, and M. Ferrari M, "A theoretical model for the margination of particles within blood vessels," Ann. Biomed. Eng., vol. 33, no. 2, pp. 179-190, 2005.

[5] P. Decuzzi and M. Ferrari M, "The adhesive strength of non-spherical particles mediated by specific interactions," Biomaterials, vol. 27, no. 30, pp. 5307-5314, 2006.

[6] P. Decuzzi and M. Ferrari, "Design maps for nanoparticles targeting the diseased microvasculature," Biomaterials, vol. 29, no. 3, pp. 377-384, 2008.

[7] M. Kojic, N. Filipovic, B. Stojanovic, and N. Kojic, Computer Modeling in Bioengineering: Theoretical Background, Examples, and Software. Chichester, U.K: Wiley, 2008.

[8] H. Hatzikirou, L. Brusch, C. Schaller, M. Simon, and A. Deutsch, "Prediction of traveling front behavior in a lattice-gas cellular automaton model for tumor invasion," Comput. Math. Appl., vol. 59, no. 7, pp. 2326-2339, 2010.

[9] G. J. Wagner and W. K. Liu, "Coupling of atomistic and continuum simulations using a bridging scale decomposition," J. Comput. Phys., vol. 190, pp. 249-274, 2003.

[10] S. Tang, T. H. Hou, and W. K. Liu, "A mathematical framework of the bridging scale method," Int.J. Num. Meth. Eng., vol. 65, pp. 1688-1713, 2006.

[11] M. Kojic, N. Filipovic, and A. Tsuda, "A mesoscopic bridging scale method for fluids and coupling dissipative particle dynamics with continuum finite element method," Comput. Methods Appl.Mech.Eng, vol. 197, pp. 821-833, 2008.

[12] A. T. Chwang and T. Y. Wu, "Hydromechanics of low-Reynolds-number flow-Part 4: Translation of spheroids," J. Fluid Mech., vol. 75, no. 4, pp. 677-689, 1976.

[13] H. Hu and D. Joseph, "Direct simulation of fluid particle motion," Theor Comput. Fluid Dyn., vol. 3, pp. 285-306, 1992.

[14] N. Filipovic, M. Ferrari, and M. Kojic, "Dissipative particle dynamics simulation of circular and elliptical particles motion in 2D laminar shear flow," Microfluidics Nanofluidics, vol. 10, no. 5, pp. 1127-1134, 2010. 